\title{
CLUSTERS Y REDES EMPRESARIALES EN EL CONTEXTO DEL TURISMO RURAL: LA EXPERIENCIA GRIEGA
}

\section{CLUSTERS AND NETWORKS IN THE RURAL TOURISM CONTEXT: THE GREEK EXPERIENCE}

\section{AUTOR}

\section{Marios Soteriades}

TEI de Creta, Heraklion, Creta (Grecia).

marsot@sdo.teicrete.gr

\section{RÉSUMÉ}

Cette intervention vise principalement à examiner les facteurs déterminant le succès de clusters et réseaux entrepris dans le domaine du tourisme rural. La bibliographie scientifique internationale suggère que les clusters et réseaux constituent des outils efficaces de gestion et de marketing. Une étude de cas a été effectuée afin d'analyser et de mettre en évidence les facteurs contribuant à la formation et au fonctionnement de trois projets réalisés en matière de tourisme rural. Différents aspects et dimensions de gestion et de marketing ont été examinés. Le résultat final consiste à déterminer les facteurs qui sont cruciaux pour la réussite et à formuler certaines recommandations/ suggestions en matière d'alliances en tourisme rural auprès de planificateurs et de gestionnaires de destination.

\section{MOTS CLÉS}

Clusters - Réseaux - Tourisme rural - Grèce - Étude de cas. 


\section{RESUMEN}

Este trabajo tiene por objeto examinar los factores determinantes del éxito de los clusters y redes de empresas en el turismo rural. La literatura científica internacional sugiere que los clusters y redes constituyen herramientas eficaces de gestión y de marketing. Se ha realizado un estudio de caso con el ánimo de analizar y destacar los factores que han contribuido a la formación y el funcionamiento de tres proyectos de turismo rural. Diferentes aspectos y dimensiones de gestión y de marketing han sido examinados. El resultado final permite determinar los factores que han sido cruciales para el éxito, así como formular ciertas recomendaciones/sugerencias en lo que respecta a las alianzas en turismo rural según los planificadores y gestores de destinos turísticos.

\section{PALABRAS CLAVE}

Clusters - Redes - Turismo rural - Grecia - Estudio de caso.

\section{ÍNDICE}

1. Introduction

2. Littérature / Bibliographie

\subsection{Concepts}

2.2. Les clusters et réseaux dans l'industrie touristique

3. L'expérience grecque: trois projets de clusters / réseaux de tourisme rural

3.1. Méthodologie et contexte de l'étude de cas

3.2. GUEST INN : un réseau de gîtes / hébergements ruraux grecs

3.2.1. Un Guest Inn: la marque commerciale et la charte de qualité

3.2.2. Les membres du réseau

3.2.3. Champ d'activités

3.3. «Terre du Psiloristis»: un cluster géographique à Anoghia, Crète

3.3.1. Profile

3.3.2. But, objectifs et activités du cluster 
3.4. «Les routes des vins de la Grèce du Nord»: un cluster géographique et thématique

3.4.1. Profile

3.4.2. Les routes des vins: but, objectifs et activités

\subsection{Résultats et Problèmes}

4. Conclusions: facteurs cruciaux et suggestions

5. Bibliographie

6. Webgrafía

7. Annexe

\section{Introduction}

Les entreprises touristiques sont confrontées à une concurrence toujours plus vive et obligées de fonctionner dans un environnement très compétitif (Spindler 2003, Hall 2008a). De plus, le marché globalisé n’implique plus des unités d'entreprises individuelles, mais il est plutôt formé de destinations géographiques ou thématiques composées d'un réseau ou d'un cluster d'entreprises impliquées dans l'activité touristique (Lazzeretti et Petrillo 2006). La littérature scientifique suggère que les clusters et réseaux pourraient être utilisés comme un cadre fournissant aux Petites et Moyennes Entreprises Touristiques (PMET) -qui ne possèdent pas les ressources ni les aptitudes organisationnelles afin de survivre avec leur propres forces- des opportunités de fonctionner dans un environnement touristique compétitif. L'évolution la plus intéressante en matière de tourisme dans les années 90 a résidé en de nouvelles formes d'alliances, qui sont fondées sur une participation commune aux domaines de planification, de gestion et de marketing des destinations touristiques (Middleton 2002). Les enjeux auxquels ont à faire face les entreprises touristiques dans les zones rurales ont été reconnus depuis longtemps par différents auteurs (entre autres Wilson et al. 2001, Sharpley 2005, Hall 2005). Le rôle vital des 
alliances proactives entre le secteur public et le secteur privé a été signalé et démontré par de nombreux auteurs (voir par exemple Middleton 2002, Fyall et Garrod 2005) et pendant une longue période, ces formes de coopération furent la méthode favorite pour affronter les problèmes des PMET et de destinations dans des conditions de mondialisation. Cependant, plus récemment, l'attention s'est progressivement portée sur la contribution des clusters et réseaux, qui sont considérés comme une solution pour engendrer des économies positives pour les entreprises touristiques et la compétitivité régionale (Porter 1998, Poon 2002). À partir du moment où les destinations et les régions deviennent plus compétitives afin d'acquérir une part de marché touristique plus grande, les clusters et réseaux deviennent à leur tour de plus en plus importants. Il en va de même pour les linkages à l'intérieur du secteur privé, à cause de la nature des produits touristiques qui ne sont qu'un amalgame des composantes multiples offertes par les diverses entreprises. On pourrait soutenir que plus le marché est mûr, plus le motif est sérieux pour les acteurs individuels de rechercher les bénéfices résultant des synergies d'alliance (Hall 2005 et 2008b).

Le but de la présente intervention est d'examiner les facteurs associés à la réussite des clusters et réseaux entrepris dans le domaine du tourisme rural. On procèdera tout d'abord à un examen sommaire de la bibliographie concernant les concepts, le contexte de destination touristique, les activités et la contribution potentielle des clusters et réseaux. Puis on présentera une étude de cas de trois projets réalisés en Grèce en matière de tourisme rural, en mettant l'accent sur les aspects principaux. Enfin, on tentera de mettre en évidence les facteurs considérés comme étant déterminants pour la réussite de clusters / réseaux. 


\section{Littérature / Bibliographie}

\subsection{Concepts}

Le tourisme est une industrie hautement diversifiée et fragmentée; il en va de même pour le tourisme rural qui en général est considéré comme incluant un large éventail de facilités touristiques: hébergement, activités de loisirs, événements, festivals, sports et recréation; toutes ces facilités sont développées en milieu rural. On pourrait suggérer que le tourisme rural constitue un concept qui comprend toute activité touristique en zone rurale (Béteille 2000, Soteriades et Varvaressos 2002). Il faut remarquer que la définition du tourisme rural doit être formulée à partir d'une perspective de l'expérience du visiteur. Certes, l'«expérience» dans une destination rurale est beaucoup plus importante que les attraits et produits spécifiques. La zone rurale et la campagne sont devenues des destinations pour une clientèle croissante, en quête d'authenticité, d'ambiance et d'environnement non endommagés (Béteille 2000). L'enjeu pour les entreprises du tourisme rural consiste à monter des produits adéquats, à un niveau de qualité tel qu'il est perçu par cette clientèle, et à les faire distribuer et commercialiser de manière professionnelle. Ainsi, le tourisme rural constitue simultanément une forme de comportement du consommateur et une stratégie par laquelle les destinations pourraient développer et commercialiser des attractions et une ambiance rurales.

Un cluster est simplement le regroupement, au sein d'une région particulière, d'entreprises ou de branches d'entreprises qui sont interconnectées par le biais de leurs produits, de leurs marchés - et d'autres entreprises ou d'organisations, telles que les fournisseurs, avec qui elles sont en interaction dynamique. Porter définit les clusters comme étant 'des concentrations géographiques d'entreprises interconnectées, de fournisseurs spécialisés, de prestataires de services, de compagnies actives en des branches connexes et des institutions associées (par exemple universités et chambres de commerce et d'industrie) dans des champs 
particuliers et qui se concurrencent mais aussi coopèrent' (Porter 1998, p. 197). Le montage de cluster est une procédure qui aide les partenaires à profiter des synergies et des complémentarités entre leurs outputs, engendrant des bénéfices divers. Un cluster est donc une forme progressive de réseau d'entreprises, ayant des buts bien déterminés et précis, centrés sur l'accroissement des ventes et I'amélioration de la profitabilité. Cette forme d'alliance rend possibles et efficaces l'échange d'information et le transfert de technologie (savoir-faire), en encourageant différents dispositifs de coordination et de collaboration dans leur cadre (European Commission 2003). Les réseaux sont décrits comme 'un type spécifique de relation unissant un ensemble d'individus, objets ou événements' et sont considérés comme un concept organisationnel pour promouvoir l'action commune (Marsh 1998). En substance, les réseaux sont caractérisés par une multitude de partenaires qui transcendent des structures et des limites organisationnelles et impliquent un engagement des partenaires membres du réseau à un ensemble de buts communs et, très probablement, le partage d'une vision et de vues globales. Les clusters et réseaux sont d'une importance vitale pour le développement régional, car ils contribuent à accroître les performances, à améliorer les capacités d'innovation et à former une masse critique d'entreprises locales.

\subsection{Les clusters et réseaux dans l'industrie touristique}

Les clusters et réseaux engendrent de nombreux bénéfices, tels que (Michael 2003, Poon 2002, Saxena 2005): des économies d'échelle, un intérêt centré sur la coopération et l'innovation, des synergies, une productivité augmentée, un transfert de connaissance, avec co-marketing et co-promotion, une compétitivité améliorée et un avantage compétitif durable; tous ces bénéfices créant des occasions de synergie et de renforcement mutuel afin d'atteindre le but principal d'une destination touristique; à savoir, offrir une expérience de vacances récompensant pleinement ses visiteurs. Les PMET ont à faire face au défi du renouvellement des modèles de gestion pour maintenir leur compétitivité en une économie mondialisée. 
La formation de clusters et réseaux, et les innovations de produit constituent des réponses typiques à ce défi (Kokkonen et Tuohino 2007). La recherche scientifique indique que la formation des réseaux est une nouvelle source d'avantages compétitifs et une exigence essentielle de gestion régionale et globale. Des études récentes (cf. par exemple Michael 2003, Hall 2005 et 2008b, Novelli et al. 2006, Saxena 2005, Tinsley et Lynch 2007) examinent plus profondément les implications de la formation des clusters et réseaux en matière de tourisme et d'hôtellerie. Michael (2003) a fait remarquer l'importance de la 'structure' et de l'échelle' des clusters, en particulier lorsqu'ils sont mis en œuvre dans le contexte du tourisme. Le même auteur insiste également sur la « création des opportunités économiques et sociales dans les petites communautés, à travers le développement de clusters d'entreprises complémentaires qui seraient en mesure de fournir collectivement un ensemble d'attributs pour former un produit régional spécialisé » (Michael 2003, p. 3). Selon Hall et Sharples (2008b) les clusters et réseaux sont reconnus comme faisant partie intégrale du succès de nombreux partenariats de tourisme alimentaire, combiné bien évidemment avec des activités et des produits de tourisme rural. Les clusters et réseaux sont à coup sûr considérés comme étant des dispositifs efficaces de gestion et de marketing pour les destinations touristiques rurales. Ces outils doivent être utilisés de manière appropriée afin de contribuer au développement touristique durable et à la réalisation des buts des entreprises connexes (Kokkonen et Tuohino 2007, Tinsley et Lynch 2007).

L'industrie du tourisme est caractérisée par une très grande hétérogénéité des activités, une inévitable diversité structurale et la prédominance des petites entreprises (Spindler 2003). Pour ces raisons, certains auteurs ont insisté sur l'idée qu'il n'existerait pas d'alternative logique au développement des alliances au niveau local impliquant des entreprises touristiques et d'autres partenaires locaux (Lazzeretti et Petrillo 2006). Vu que par le biais d'un cluster, un groupe de PMET peut devenir 
compétitif sur le plan global en coopérant localement ${ }^{1}$, des réseaux et clusters dans le tourisme ont connu une croissance considérable, apportant des bénéfices tels que le partage des informations de marketing, l'innovation, l'opportunité d'entrer dans d'autres clusters à un niveau national et transnational, le développement des ressources et du transfert de connaissances entre partenaires / co-actionnaires (Saxena 2005). Le but des clusters et réseaux touristiques consiste à mettre en évidence la disponibilité de certaines activités au sein d'une destination et d'unir les PMET qui, autrement, fonctionneraient et agiraient isolément, afin de coopérer et de monter un produit touristique local durable et profitable à l'ensemble de l'économie locale. C'est précisément à cause de cette fragmentation que tous les acteurs participant à la chaîne de valeur doivent traiter des enjeux tels que l'intégration, la collaboration, la formation de clusters et réseaux pour leurs activités (Poon 2002). De nos jours, les touristes s'attendent à une gamme de services qui leur permette des options multiples et des formules offrant des occasions d^expériences'. La chaîne de valeur d'une destination constitue ainsi l'image de tous ses éléments. Cette approche nécessite la coopération et la formation de réseaux entre les acteurs-clés. Lemmetyinen et Go (2009) suggèrent que le développement de réseaux d'entreprises touristiques pourrait être envisagé comme un système dans lequel tout participant contribue avec ses propres capacités, aptitudes et compétences. Les mêmes auteurs mettent l'accent sur l'importance d'une approche de réseau pour s'engager et gérer dans une participation active; ils suggèrent donc que les entreprises touristiques locales doivent développer de nouvelles aptitudes et capacités-clés afin de faire face à une concurrence globale sévère. Ainsi, la coordination des activités communes dans les réseaux d'entreprises touristiques est envisagée comme une condition préalable pour conforter la procédure de création de valeur et la formation d'une procédure d'identité de marque commerciale au sein du cluster. Il en va de même pour l'enjeu de la qualité ; des auteurs (voir par exemple Canard et Ravix 2003) suggèrent qu'il convient d'adopter une approche plus large qui

\footnotetext{
${ }^{1}$ A cet égard, il serait utile de reprendre les propos ou l'aphorisme mémorable de Naisbitt (1994, p. 24) 'think local ; act global', cité par Middleton et Hawkins (2002, Pág. 130).
} 
intègre la qualité des entreprises et des destinations, d'où le développement de relations partenariales qui englobent préoccupations économiques, sociales mais aussi environnementales, tout en restant centrées sur la fonction première d'une démarche de qualité, à savoir la satisfaction du consommateur. L'option pour une approche de cluster est en outre justifiable par le fait que celui-ci peut constituer un des meilleurs outils disponibles pour soutenir le développement touristique durable. Une autre étude (Kokkonen et Tuohino, 2007) a analysé les procédures d'innovation et la dynamique de formation des réseaux. Les auteurs ont suggéré que l'innovation dans les réseaux de PMET est une procédure complexe et synthétique consistant en des innovations de produit, de procédure et des ressources.

La contribution significative des clusters et réseaux touristiques a été analysée et mise en évidence dans des contextes touristiques divers, tels que la formation de réseau régional et virtuel: I'industrie du tourisme existe et fonctionne comme un réseau de sous-branches interconnectées et, par conséquent, le montage de réseaux est très bénéfique en matière de marketing de destination (Hitz et al. 2006, Soteriades et Avgeli 2007). Les Technologies de I'Information et de la Communication (TIC) contribuent à l'efficacité des clusters d'entreprises et encouragent une collaboration plus étroite. Les destinations semblent être les bénéficiaires majeurs des TIC, dans la mesure où elles peuvent profiter d'un nouvel dispositif stratégique pour la planification, la gestion et le marketing à travers la coordination, et d'un pouvoir accru en canaux de distribution. Ces bénéfices pourraient être atteints moyennant une alliance plus étroite et plus solide au sein de l'industrie du tourisme. On peut trouver des réseaux à l'intérieur des organisations, entre les entreprises et leurs partenaires, aussi bien qu'entre l'ensemble des individus et des entreprises. Des synergies et une interopérabilité entre des partenaires extérieurs et l'ensemble des opérations aident les entreprises à réduire leurs coûts de travail, à améliorer leur efficacité, à pratiquer une différenciation flexible et à soutenir leurs procédures de gestion (Buhalis 2005). L'Internet permet la création d'entreprises virtuelles dans lesquelles les TIC fournissent des linkages, en particulier 
des réseaux pour les micro-entreprises. Il est suggéré que les PMET pourraient bénéficier de flux d'information accrus à travers les clusters régionaux, pour faciliter leur visibilité commerciale, leur positionnement global et obtenir un levier stratégique (Hitz et al. 2006). Le développement du portail touristique www.purenz.com constitue un exemple réussi des alliances multilatérales et des initiatives communes et concentrées qui sont requises afin de promouvoir et commercialiser la Nouvelle Zélande comme destination touristique (Bhat 2004). En appliquant de telles méthodes basées sur le Web marketing, Tourism New Zealand a été très efficace pour obtenir le meilleur résultat possible avec un budget de promotion restreint. Les réseaux sont fondamentaux, non seulement pour engendrer des opportunités de promotion et de marketing de l'industrie locale, mais aussi pour créer les conditions préalables nécessaires afin d'innover le produit régional. La littérature sur la gestion d'événements (cf. par exemple Getz et al. 2007 et Bernini 2009) a reconnu l'importance de la création de relations avec d'autres acteurs dans le contexte du tourisme événementiel. L'étude de cas de Lismore en Australie (Mackellar 2006) démontre que les activités de festival, telles que les concours de recettes de cuisine et les démonstrations de chefs renommés, permettent aux agriculteurs locaux et aux visiteurs de découvrir de nouvelles interconnections entre la gastronomie et d'autres branches productives. On a émis l'hypothèse que (a) un réseau digne de confiance et ayant des relations stables à long terme permet la diminution de l'incertitude opérationnelle et, par conséquent, l'amélioration de l'efficacité intérieure, (b) un réseau opérant sans collaboration, c'est-à-dire ne fonctionnant pas vers des buts et objectifs mutuellement désirables, aboutit à une inefficacité interne. Stokes (2006) a examiné les réseaux inter-organisationnels qui influencent la stratégie du tourisme événementiel arrêtée par les agences de nature mixte (publique et privé) développant des événements en Australie. Il a suggéré que le développement du tourisme événementiel mené par les agences australiennes compétentes en la matière est guidé essentiellement par des stratégies de nature proactive et réactive. Ces agences mixtes animent et dirigent des réseaux flexibles et informels qui consistent en des clusters relativement stables et composés de membres intra- 


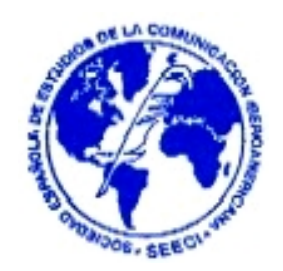

gouvernementaux et d'entreprises. Le tourisme vignoble a été cité comme un exemple de développement réussi de clusters (Hall 2005 et 2008b). Selon Getz et Brown (2006) la collaboration est nécessaire afin de faciliter l'expérience du tourisme vignoble, impliquant des organismes de gestion / marketing de destination, des viticulteurs, des vignerons, des professionnels du tourisme et des prestataires des services de culture et de loisirs. Un cluster fonctionnant dans le domaine du tourisme de spa et de santé a été analysé par Novelli et al. (2006). L'expérience de « Healthy Lifestyle Tourism Cluster » britannique a été étudiée afin d'examiner la procédure et les implications de la formation, ainsi que le fonctionnement d'un cluster touristique. Selon les auteurs, ce projet a démontré que les responsables doivent porter leur attention sur la procédure plutôt que sur les résultats. Cependant, la formation de clusters ne devrait pas être envisagée comme une procédure simple et spontanée, en raison de la nature des entreprises impliquées, mais comme une procédure complexe reliée à une collaboration étroite et vigoureuse des partenaires.

L'industrie du tourisme est avant tout centrée sur l'information et la satisfaction des consommateurs (Canard et Ravix 2003, Soteriades et Avgeli 2007). Étant donné que les touristes sont toujours en quête d'une expérience de vacances émouvante, gratifiante et diversifiée, les efforts doivent être dirigés vers l'identification des opportunités et possibilités de synergie et de renforcement mutuel. La réussite d'une destination en termes de satisfaction des visiteurs est fonction de plusieurs composantes interdépendantes. Par conséquent, les activités de clusters et réseaux sont déployées principalement dans deux domaines, à savoir: (a) Gestion: la formation de clusters / réseaux contribue à renforcer des linkages économiques en amont, afin de réduire les fuites, de faciliter la collaboration, le développement de l'appréhension et de la connaissance du tourisme par la population locale, des actions et activités de gestion et de partage de savoir et d'informations, échange de renseignements et d'expertise, innovations et suivi des résultats obtenus et adaptation, si nécessaire, des produits et des modalités de gestion ; (b) Marketing: cette fonction fournit des techniques et des méthodes pour communiquer des 
informations, adapter les produits, influencer les touristes et exercer un suivi sur le comportement des consommateurs (Fyall et Garrod 2005). Toutes les variables du marketing mixte, les outils et les techniques de communication pourraient aisément être influencés d'une manière positive par la synergie produite à travers leur coordination au sein d'une seule équipe de management: développement et montage de produits (chaîne de valeur et innovation), enquêtes et études de marché, segmentation du marché, branding et formation d'une image commerciale, promotion, services d'information, banque de données de clientèle et marketing relationnel. Des nombreux auteurs (cf. par exemple Buhalis 2005, Hitz et al. 2006) ont fait remarquer la contribution des TIC en matière. Les TIC disponibles peuvent stimuler la formation de clusters et réseaux, et de nouvelles formes de coopération entre les entreprises. Les réseaux électroniques d'entreprises ont la capacité d'unir les entreprises et les partenaires au sein d'une alliance locale ou régionale afin de surmonter certains problèmes.

Pendant les années 90 et la décennie actuelle, des projets de clusters et de réseaux ont été mis en œuvre partout en Europe. Dans ce contexte général, une série d'initiatives ont été entreprises et des projets ont été réalisés dans le domaine du tourisme rural en Grèce. Ces projets visent principalement à renforcer la compétitivité et à soutenir l'efficacité des entreprises du tourisme rural. Dans la section suivante sont analysés trois de ces projets. La méthodologie d'étude de cas a été appliquée afin d'examiner et de mettre en évidence les facteurs contribuant à la réussite de clusters et réseaux.

\section{L'expérience grecque: trois projets de clusters / réseaux de tourisme rural}

\subsection{Méthodologie et contexte de l'étude de cas}

La présente étude a été réalisée suivant la méthodologie d'étude de cas qualitative. Il faut souligner que cette méthodologie permet d'examiner un phénomène en 
obtenant des explications relatives possibles (Yin 2003). Notre étude s'est basée sur la consultation des documents d'information et de promotion des projets en question. En outre, des entretiens semi-structurés ont été effectués auprès des coordinateurs-animateurs des trois projets. Notre but était donc d'apporter des précisions sur les meilleures pratiques et sur les facteurs déterminant la réussite en matière de clusters et réseaux en tourisme rural.

Beaucoup de communes et de zones rurales sont en concurrence avec un nombre croissant de régions qui s'efforcent d'entrer sur le marché touristique. Il semble que la seule voie pour faire avancer et mener à bien cette tâche consiste à identifier et à monter un portefeuille d'activités de loisirs en plein air et à l'intérieur. Il est donc vital de procéder à un échange d'idées, de connaissances et de savoir-faire, et de diversifier les produits et les prestations offerts (Soteriades etet Varvaressos 2002). On estime que le meilleur moyen pour atteindre ce but, et probablement celui qui a le meilleur rapport coût - résultat et qui permet ainsi de demeurer compétitif, passe par le renforcement des réseaux existants et par la formation de clusters nouveaux, au sein desquels connaissances, expertise et idées innovatrices pourraient s'échanger afin former un portefeuille de tourisme rural bien distinct et durable (Wilson et al. 2001, Hall 2008a). Dans ce cadre, il semble très intéressant d'examiner les facteurs et les dimensions de réseaux et clusters qui ont été mis en œuvre afin d'en tirer certaines leçons, notre but étant d'arriver à formuler des suggestions pour les planificateurs et les gestionnaires de destinations. Pour ce faire, trois projets réalisés en Grèce ont été examinés: (1) Le réseau d'hébergements / gîtes ruraux Guest Inn (2) un cluster géographique intitulé 'Terre du Psiloritis' en Crète ; et (3) les 'Routes des Vins', un cluster géographique et thématique de tourisme rural.

Les trois clusters / réseaux ont été analysés par rapport aux caractéristiques suivantes: (a) acteurs: volume et type des acteurs impliqués, leurs besoins et attentes; (b) activités: gestion et marketing; (c) structure: taille; type de structure d'organisation; et (d) résultats et problèmes. 


\subsection{GUEST INN: un réseau de gîtes / hébergements ruraux grecs}

Guest Inn est un réseau grec de gîtes ruraux, membre de la Fédération Européenne des Gîtes (www.eurogites.org). Le réseau a commencé à fonctionner en 2003 sous une structure formelle, comme société personnelle (partenariat général). Sa mission consiste à présenter et à promouvoir, via internet, de petites unités d'hébergement, ainsi qu'à formuler des suggestions aux voyageurs et à fournir des renseignements sur les gîtes qui sont conformes à des standards de qualité communs, tels que la chaleur de l'accueil, le confort du séjour, le caractère soigné et fonctionnel de l'architecture et I'originalité particulière de l'ambiance. Le réseau concerne tout d'abord les propriétaires de gîtes ruraux remarquables, qui d'habitude sont situés dans des régions non envahies par le tourisme organisé, ne sont très commercialisés par les agences de voyages et désirent attirer des visiteurs potentiels tout au long de l'année. En deuxième lieu, il s'adresse également aux touristes grecs et étrangers s'intéressant à connaître des villages et des endroits peu fréquentés, et à passer leurs vacances en Grèce, hors des sentiers battus. Les établissements appartenant au réseau et recommandés aux touristes sont situés dans un environnement authentique et une ambiance tranquille et sont bien entretenus, dans le respect de l'architecture locale.

\subsubsection{Un Guest Inn : la marque commerciale et la charte de qualité}

Le but principal du réseau est d'établir une marque commerciale (branding), qui pourrait servir de dispositif pour la distribution et la commercialisation des gites ruraux. Cette marque pourrait également être utilisée comme un outil de gestion, c'est-à-dire aider à se conformer à des normes opérationnelles et de qualité (Chaspoul 2000, Canard et Ravix 2003). La méthode la plus efficace en termes de coûts et de temps pour créer une certaine forme d'identité commerciale visible ne pouvait être autre que la création d'un logotype (Figure 1) et le montage d'un site 
internet (www.guestinn.com). Ce logotype constitue la base pour la marque potentielle.

Figure 1. Le logotype du réseau Guest Inn

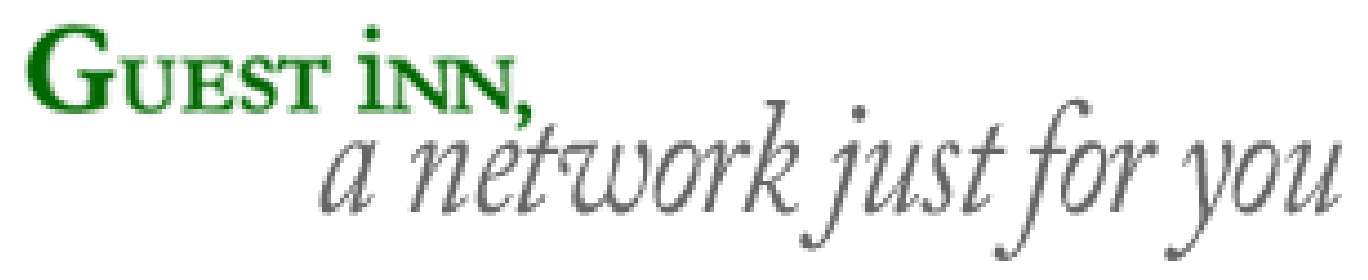

Les unités d'hébergement qui deviennent membres du réseau sont sélectionnées indépendamment de leur catégorie et de leur tarifs, mais à une seule condition; qu'elles satisfassent certains critères de qualité préétablis (cf. Tableau 1). Ainsi, le sigle Guest Inn est une garantie de qualité pour les touristes. Une classification à base $d^{\prime}$ «héliotropes» (1, 2 ou 3 soleils) détermine la catégorie de I'hébergement et aide les consommateurs à trouver celui qui correspond le mieux à leurs besoins et désirs. Le but principal du réseau est donc de faire de sa marque commerciale une garantie de qualité des prestations. Cette démarche favorise ainsi l'instauration de relations «partenariales » entre professionnels qui leur permettent d'innover et d'élargir la gamme de leurs produits et de leurs prestations (Canard et Ravix 2003).

Tableau 1. Réseau Guest Inn - Critères de qualité

\begin{tabular}{|c|l|}
\hline $\begin{array}{c}\text { Domaine / } \\
\text { Champ }\end{array}$ & \multicolumn{1}{c|}{ Critères } \\
\hline $\begin{array}{c}\text { Hospitalité / } \\
\text { réception }\end{array}$ & $\begin{array}{l}\text { Attention personnelle et accueil chaleureux dispensés par les } \\
\text { propriétaires eux-mêmes. }\end{array}$ \\
\hline Séjour & $\begin{array}{l}\text { Décor agréable, propreté impeccable, confort, équipements en } \\
\text { bon état. Petit déjeuner traditionnel, préparé à la maison. }\end{array}$ \\
\hline Architecture & $\begin{array}{l}\text { Petites unités hébergées dans des bâtiments nouveaux ou } \\
\text { rénovés, dans tous les cas en harmonie avec l'environnement et } \\
\text { l'architecture de chaque région. }\end{array}$ \\
\hline Environnement & $\begin{array}{l}\text { Paysages d'une beauté naturelle exceptionnelle, offrant au } \\
\text { visiteur paix, sérénité et repos, ainsi que l'occasion de connaître } \\
\text { la campagne grecque et ses produits de terroir, d'entrer en } \\
\text { contact avec les habitants et leurs traditions. }\end{array}$ \\
\hline
\end{tabular}

Source: www.guestinn.com/en/quality 


\subsubsection{Les membres du réseau}

Les destinations recommandées sont loin des sentiers battus et du tourisme organisé, ce sont des régions authentiques de la Grèce inconnue, offrant aux visiteurs l'occasion de découvrir l'hospitalité hellénique traditionnelle et la beauté de la campagne grecque. La répartition spatiale et par type d’hébergement est présentée dans le Tableau 2.

Tableau 2. Les membres du Guest Inn - Répartition par région et type d'hébergement

\begin{tabular}{|l|c|c|c|c|c|c|}
\hline \multirow{2}{*}{ REGION } & \multirow{2}{*}{ Unités } & \multicolumn{5}{|c|}{ TYPE } \\
\cline { 3 - 7 } & & $\begin{array}{c}\text { Demeures } \\
\text { et Gîtes } \\
\text { ruraux }\end{array}$ & Meublés & Hôtels & $\begin{array}{c}\text { Auberg } \\
\text { Chambre } \\
\text { s d'hôte }\end{array}$ & \\
\hline Macédoine & 7 & & & 1 & & 6 \\
\hline Thrace & 3 & 1 & & 2 & & \\
\hline Épire & 7 & 1 & & 1 & & 5 \\
\hline Thessalie & 4 & & 1 & & & 3 \\
\hline Grèce centrale & 2 & & & & 1 & 1 \\
\hline Péloponnèse & 10 & 1 & 2 & 1 & 1 & 5 \\
\hline Îles ioniennes & 5 & 4 & & & & 1 \\
\hline Egée nord-est & 5 & 3 & 1 & & & 1 \\
\hline Cyclades & 8 & 1 & 4 & 2 & & 1 \\
\hline Crète & 9 & 8 & & & & 1 \\
\hline TOTAL & 60 & 19 & 8 & 7 & 2 & 24 \\
\hline Distribution \% & $100 \%$ & $31,7 \%$ & $13,3 \%$ & $11,7 \%$ & $3,3 \%$ & $40 \%$ \\
\hline
\end{tabular}

Source: http://www.guestinn.com/en/allpans.php

\subsubsection{Champ d'activités}

Il semble que l'œuvre principale du réseau est la formation d'une chaîne volontaire ${ }^{2}$ avec une marque commerciale (label). Une marque collective commune pourrait être très profitable à un meilleur positionnement sur le marché; à des actions communes de marketing et de promotion; à une distribution efficace (par biais d'un système de distribution centrale); à des normes opérationnelles et de gestion. Enfin, afin

\footnotetext{
${ }^{2}$ Une chaîne volontaire est une forme de consortium d'unités d'hébergements. Les établissements membres de la chaîne demeurent propriété des entrepreneurs - partenaires, quoiqu'ils unissent leurs forces pour s'assurer de meilleures prestations de gestion et de marketing. En principe, la chaîne est constituée sous forme d'association ou d'entité juridique. Par application de ses statuts, les membres s'engagent à observer une charte intérieure. Citons deux exemples: Mapotel et Logis de France.
} 


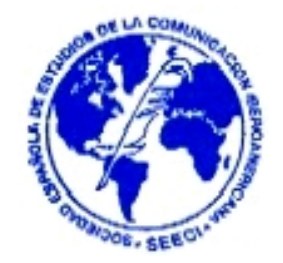

d'enrichir le séjour et de le rendre plus agréable, d'autres activités de loisir et récréatives sont offertes sur place et fournies via le réseau, telles que canyoning, kayak, rafting, randonnées pédestres, escalade, balades à cheval et à vélo, visites des domaines et dégustation de vin, sports nautiques, plongée, observation d'oiseaux, ski et snow-board.

Le website du Guest Inn fournit des informations relatives au réseau et d'autres renseignements généraux. Le réseau offre également des possibilités de partenariat. Le réseau est affilié à la compagnie maritime Minoan Lines, qui offre un rabais de $20 \%$ sur présentation du bon d'échange à tous les clients ayant fait leur réservation on-line sur le www.guestinn.com.

Figure 2. Le banner publicitaire du Guest Inn

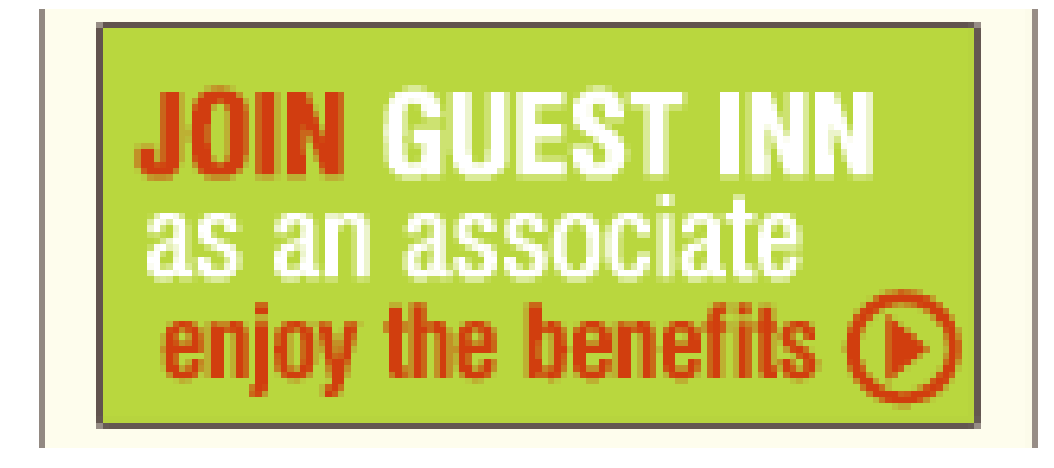

\section{3 «TERRE DU PSILORITIS »: un cluster géographique à Anoghia, Crète}

\subsubsection{Profile}

Le cluster «Terre du Psiloritis» a été créé par des entreprises, agences et associations partageant un but commun; à savoir la sauvegarde, le développement et la promotion de l'identité, de l'authenticité spéciale de la zone géographique (montagne centrale de l'île, cf. la carte no 1 en Annexe), par le biais d'actions concertées centrées sur le montage et la promotion des produits de tourisme rural (www.idinet.gr). Le cluster a été fondé en 2005 sous la forme juridique d'une société 
à responsabilité limitée (SARL). Les partenaires sont des entreprises provenant de différentes branches productives complémentaires: hébergements ruraux, restauration-cafés, exploitations agricoles, agro-alimentaire, commerçants et associations culturelles. Ce projet a reçu un apport financier (pour certaines dépenses opérationnelles) dans le cadre du programme d'action local de l'initiative européenne Leader+. Les partenaires du cluster sont au nombre de 44 (voir Tableau $3)$.

Tableau 3 : Les membres du cluster Terre du Psiloritis

\begin{tabular}{|l|c|c|}
\hline \multicolumn{1}{|c|}{ Branche / Activités } & \multicolumn{2}{c|}{ Partenaires } \\
\cline { 2 - 3 } & Nombre & Ventilation en \% \\
\hline Restauration - Cafés & 11 & $25,0 \%$ \\
\hline Hébergement & 12 & $27,3 \%$ \\
\hline Agences de voyages & 1 & $2,3 \%$ \\
\hline Agro-alimentaire & 14 & $31,7 \%$ \\
\hline $\begin{array}{l}\text { Artisanat, d'art } \\
\text { populaire, poterie }\end{array}$ & 5 & $11,4 \%$ \\
\hline Associations culturelles & 1 & $2,3 \%$ \\
\hline Total & 44 & $100,0 \%$ \\
\hline
\end{tabular}

\subsubsection{But, objectifs et activités du cluster}

Le but principal consiste en la formation d'une identité commerciale et d'une marque de qualité pour les produits et services locaux. Cette tâche est mise en œuvre avec la coopération de I'AKOMM (I'agence locale de développement), l'apport financier du programme d'action local de Leader+, et le transfert du savoir-faire de la coopération transrégionale. L'expérience accumulée au sein du réseau local et interrégional a permis et facilité l'adoption et l'introduction d'une plateforme d'intérêts partagés et de bénéfices mutuels. Ainsi, il semble que le cluster s'avérerait très utile et profitable 
au développement durable et à la gestion des entreprises de tourisme rural. L'objectif principal du cluster consiste donc à pourvoir d'une expression concrète et d'une forme entrepreneuriale toutes les activités productives au sein de la région du Psiloritis, en déployant des actions telles que: fourniture de conseils techniques et de services de conseil à ses partenaires (certification de qualité, spécifications et normes, activités de marketing et études / enquêtes de marché); promotion de produits locaux ; marketing et commerce électroniques, salons et expositions professionnelles, développement et montage des produits; clustering avec d'autres partenaires locaux; et enfin mise en application des activités et projets novateurs.

Le site internet du cluster est utilisé comme un tableau d'information et d'annonces pour des événements à venir. Il s'efforce de devenir davantage un outil commercial au service des consommateurs, enregistrant itinéraires, événements, hébergements, gastronomie locale et prestataires de services et de loisirs disponibles dans la région. Un des résultats principaux du cluster est le label commercial local (brand name) 'Psiloritis Crète', qui s'inspire des bergeries saisonnières dites mitata, sortes de burons que l'on rencontre uniquement sur le mont Psiloritis.

Figure 3. Psiloritis brand name

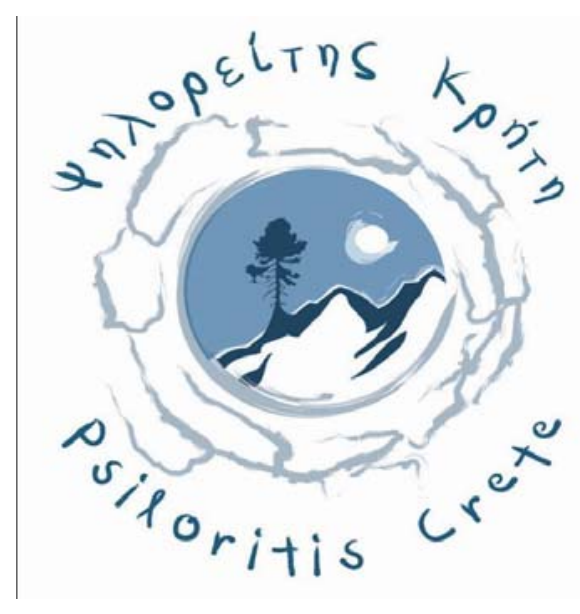

Ce label est une composante de la charte de qualité signée entre l'agence de développement d'Héraklion SA et le cluster 'Terre du Psiloritis'. Les aspects 
économiques des actions attachées à ce label sont: (a) de signaler les produits offerts et les services fournis dans la région du Psiloritis et ayant une valeur particulière; (b) de faciliter la collaboration des agences locales et des professionnels, en vue d'un développement et d'une gestion durables ; et (c) établir un pont entre la production locale et la gastronomie.

\section{4 «Les routes des vins de la Grèce du Nord»: un cluster géographique et thématique}

Il s'agit d'un partenariat interrégional conclu entre l'Association des Producteurs de Vin du Vignoble de la Grèce du Nord, quinze agences de développement local et une collectivité locale (www.wineroads.gr). L'association des vignerons a été constituée en 1993 en coopérative par les efforts communs des treize membres initiaux. Son but était de soutenir la tradition du vin et de la viticulture et d'offrir aux visiteurs l'occasion de découvrir les vignobles de la Grèce du nord. En 2002, les vignerons d'Epire et de Thrace ont rejoint l'association qui a alors changé de titre, tout en adoptant la marque commerciale et le sigle «Routes des Vins de la Grèce du Nord» (cf. la carte no 2 en Annexe). Aujourd'hui, 45 vignerons ont réuni leurs forces au sein de l'association et ont dirigé leurs activités vers un ensemble d'objectifs communs, tels que: (a) Mener une action coordonnée pour promouvoir efficacement les vins régionaux, (b) Bâtir l'image des vins de la région et les faire connaître sur le marché domestique et à l'étranger, (c) Offrir aux visiteurs un produit à thème axé autour des vignes, des paysages et des attraits touristiques de la région (d) Aider le patrimoine culturel grec, en centrant principalement sur la viticulture, le vin et les activités culturelles connexes, (e) Participer à l'élaboration de normes générales réglant les relations professionnelles entre les viticulteurs, les vignerons et les négociants en vins, afin d'optimiser la coopération, de mieux servir les consommateurs et d'améliorer la qualité des produits et des prestations de services. 


\subsubsection{Profile}

Des collectivités locales, associations culturelles, entreprises locales et autres instances sont énergiquement impliquées et participent à des activités liées aux objectifs ci-dessus (par ex. signalisation, musées folkloriques centrés sur la tradition de vin, gastronomie locale, publications et organisation des événements culturels). Le projet «Routes des Vins de la Grèce du Nord» consistait initialement en un réseau touristique pionnier en Grèce et en une manière nouvelle, pour tous les visiteurs, de découvrir les domaines ouverts au public et d'explorer les vignobles de la région, en conjonction avec des activités culturelles locales. En 2007 le réseau s'est élargi afin de comprendre des entreprises sélectionnées, telles qu'hôtels, restaurants, artisanats et fabricants de produits locaux, et prestataires de loisirs de plein air. Il est ainsi devenu un cluster, une alliance et un cadre de soutien collectif pour le vignoble et la gastronomie grecs. Le cluster a reçu un apport financier dans le cadre du programme Leader plus. Cette alliance interrégionale a pour territoire d'application toute la région nord de la Grèce et concerne la création et le développement d'un produit complet de tourisme rural ; c'est-à-dire un circuit à thème ayant comme idée centrale le vignoble. Le cluster consiste à relier, d'une part, les professionnels de I'hôtellerie, de la restauration et des producteurs de produits locaux dispersés en toute l'étendue de la région, et d'autre part, les vignobles des domaines / fabriques de vin visitables. Tous les établissements partenaires du cluster ont été sélectionnés sur la base de critères qualitatifs spécifiques et portent ainsi un label spécial de recommandation. Une image sommaire des partenaires est présentée dans le tableau suivant. 
Tableau 4 - Les partenaires du cluster 'Routes des Vins'

\begin{tabular}{|c|c|c|c|c|c|c|c|}
\hline \multirow{2}{*}{$\begin{array}{l}\text { Région } \\
\text { Branche }\end{array}$} & \multirow{2}{*}{$\begin{array}{l}\text { Macédoine } \\
\text { centrale }\end{array}$} & \multirow{2}{*}{$\begin{array}{l}\text { Macédoine } \\
\text { ouest }\end{array}$} & \multirow{2}{*}{$\begin{array}{l}\text { Macédoine } \\
\text { orientale }\end{array}$} & \multirow[t]{2}{*}{ Épire } & \multirow[t]{2}{*}{ Thessalie } & \multicolumn{2}{|l|}{ TOTAL } \\
\hline & & & & & & Nombre & en $\%$ \\
\hline Vignobles & 20 & 11 & 6 & 3 & 5 & 45 & 26,9 \\
\hline $\begin{array}{l}\text { Restaurants - } \\
\text { Cafés }\end{array}$ & 25 & 14 & 6 & 3 & 1 & 49 & 29,4 \\
\hline Hébergements & 21 & 11 & 8 & 10 & 1 & 51 & 30,5 \\
\hline $\begin{array}{l}\text { Fabricants de } \\
\text { produits locaux } \\
\text { (agro- } \\
\text { alimentaire et } \\
\text { artisanat) }\end{array}$ & 9 & 6 & 4 & -- & -- & 19 & 11,4 \\
\hline $\begin{array}{lr}\text { Activités } & \text { de } \\
\text { recréation } & \text { et } \\
\text { loisirs } & \end{array}$ & 1 & 1 & -- & 1 & --- & 3 & 1,8 \\
\hline TOTAL & 76 & 43 & 24 & 17 & 7 & 167 & 100 \\
\hline
\end{tabular}

\subsubsection{Les routes des vins : but, objectifs et activités}

Le but principal du cluster est le montage des produits ruraux à thème pour attirer des visiteurs et leur faire découvrir les vins et vignobles du nord de la Grèce. Il s'agit donc de capitaliser les investissements réalisés, les activités déjà déployées et les projets particuliers mis en œuvre; c'est-à-dire de tracer des itinéraires nouveaux et d'élargir les routes des vins montées dans le cadre de l'initiative européenne Leader II. Ces circuits à thème associent les domaines et caves coopératives visitables aux sites et attractions touristiques, ainsi qu'à des établissements de prestations touristiques, afin de valoriser le potentiel existant, de produire une valeur additionnelle pour les professionnels et de rendre la région plus attractive aux visiteurs potentiels. 
Le concept est bien connu et bien établi en France et en Europe. En fait, ces circuits sont des itinéraires touristiques proposés aux voyageurs intéressés à visiter cette région grecque. Une signalisation tout au long des routes conduit les visiteurs aux vignes, aux fabriques de vins, à des ateliers et magasins de produits et de spécialités culinaires et à d'autres curiosités touristiques (sites archéologiques, musées, monuments historiques). Des activités sont proposées simultanément: dégustation de vins, rencontre avec des vignerons, essai de produits traditionnels du terroir et de la gastronomie locale. Il s'agit donc d'une expérience des goûts, des saveurs et des connaissances taillée sur mesure, flexible, pour chaque visiteur. Les 8 routes de vins proposées par le cluster sont: Dieux d'Olympe, Épire, Naoussa, Pella - Goumenissa, Lacs, Thessalonique, Dionysos et Chalcidique. Les circuits durent 3 ou 4 jours et permettent aux visiteurs de visiter des vignobles à leur guise. En organisant et en animant des événements de recréation, de loisirs et de promotion, le cluster joue un rôle important dans le soutien du vin, élément-clé de l'identité culturelle grecque.

Le cluster participe à d'autres activités telles que: (a) Signalisation et panneaux d'information des circuits à thème dans le vignoble de la région. (b) Formation continue: organisation de séminaires auprès des professionnels et du personnel des métiers liés au vin (restaurateurs, hôteliers, guides, etc.). (c) Participation à des organismes, des associations et des agences internationales ayant des buts similaires. (d) Organisation d'événements et de colloques gastronomiques et culturels visant à familiariser les visiteurs à la tradition culinaire et au vignoble de la Grèce. Un tel événement de dégustation de vin, intitulé VorOina, a lieu chaque année dans les plus grandes villes grecques et constitue une excellente occasion pour les professionnels de présenter leurs nouveaux produits. (e) Participation à des expositions professionnelles internationales en Grèce et à l'étranger. (f) Organisation d'un concours international annuel de vins, seul en Grèce, dont le but est de présenter et de promouvoir la qualité de la production viticole auprès des professionnels et du grand public. 


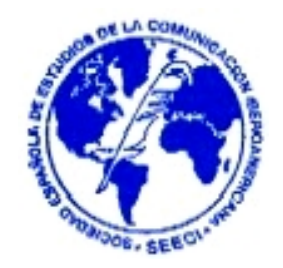

\subsection{Résultats et Problèmes}

La formation de clusters et réseaux a ses racines dans le désir de créer un ensemble plus viable et plus durable d'opportunités touristiques. Les bénéfices émanant du clustering sont: le partage des idées, le transfert de savoir à travers l'échange d'expertise et des ressources; l'amélioration des aptitudes et capacités (par exemple, les pratiques de service); l'adoption d'un système d'assurance de qualité, l'établissement d'interactions entre la production locale et la gastronomie, un marketing efficace.

Les projets examinés ont, semble-t-il, trois traits principaux (Roberts et Hall 2001, Soteriades et Varvaressos 2002): (a) les partenaires ont des buts communs et des bénéfices mutuels; (b) chaque projet crée entre les partenaires des synergies relatives aux activités accomplies au niveau local et régional; et (c) il facilite les actions précédentes. Dans le cadre de ce clustering, les activités mises en place relèvent de deux champs, à savoir la gestion et le marketing. Plus particulièrement, une série de produits à thème (p.e. routes des vins et circuits / randonnées gastronomiques) ont été développés. Ces produits incluent donc une association entre la culture (monuments et histoire), les activités agricoles, les produits locaux (vin) et la gastronomie locale, afin de produire des synergies. Un des principaux résultats des clusters est donc l'adoption et la mise en œuvre de procédures innovatrices concernant la promotion des entreprises du tourisme rural, l'amélioration des prestations de services, et la capacité et les potentialités de gestion de la demande comprenant: transfert de connaissance, échange d'expérience et d'informations, et co-marketing. Dans l'optique des entreprises impliquées, les trois projets de réseaux / clusters brièvement analysés ici semblent avoir produit des bénéfices significatifs pour les PMET qui d'habitude fonctionnaient isolément et qui désormais coopèrent et collaborent avec d'autres entreprises locales. Leurs alliances ont engendré une meilleure qualité de service et une visibilité commerciale croissante. De surcroît, elles ont créé des synergies: la collaboration commerciale est devenue un des outputs les plus apparents, aussi bien que les arrangements 


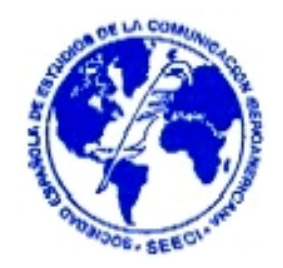

volontaires de recommandation et co-marketing via leur banner électronique. À travers le réseau, les entreprises rurales sont encouragées à fonctionner sous une forme progressive de réseau d'entreprises (cluster) ayant deux buts essentiels ; à savoir, atteindre leurs propres objectifs (amélioration des ventes et du profit) et offrir une expérience de visite améliorée (au bénéfice de leurs clients / visiteurs).

Cependant, les problèmes ne manquent pas, loin de là. La nature fragmentée et la prédominance de petites entreprises dans l'industrie touristique locale, ainsi que les faibles liens partenariaux entre les acteurs, font qu'il est très difficile au cluster touristique de rester autonome et de s'autofinancer. Les clusters offrent une certaine solution à des besoins immédiats des entreprises participantes; cependant la difficulté réside dans la tâche de maintenir la motivation des partenaires pour les objectifs à long terme (à savoir la croissance et les bénéfices économiques). Il semble très souvent que les résultats à court terme l'emportent sur les perspectives à long terme (Novelli et al. 2006). On a constaté également un autre problème, celui de la rétention des aptitudes et de la qualité de la chaîne locale de valeur. L'industrie locale est caractérisée par des interrelations faibles et instables. Dans la mesure où le but principal du cluster est de produire un chiffre d'affaires et une diversification commerciale, la chaîne de valeur doit être établie et enrichie. Les coordinateurs et animateurs des clusters / réseaux essaient coûte que coûte d'entretenir l'intérêt des partenaires et de les faire contribuer aux activités communes, ce qui souligne en quelque sorte l'importance d'un effort commun. L'expérience montre également qu'un des enjeux principaux était d'encourager les entreprises locales à envisager les implications à long terme du développement économique plutôt que les bénéfices à court terme. D'autres problèmes soulevés sont les suivants: le manque de leadership et d'engagement partagé, ainsi que l'absence d'enthousiasme. Il est impératif d'avoir une organisation centralisée ou un leadership musclé, afin de renforcer davantage les liens partenariaux entre les membres. Les clusters manquent d'informations sur le marché afin de se fixer et de suivre des objectifs réalistes, la plupart ne coordonnent pas de programmes de gestion de visiteurs et $n^{\prime} i m p l i q u e n t$ pas les entreprises locales 
dans la procédure de fixation et de réalisation des objectifs convenus. Un dernier problème, mais pas le moindre, est que les projets examinés n'exploitent pas pleinement les outils et les possibilités fournis par les TIC, essentiellement le logiciel Web 2.0 (de communication et de networking social). Les clusters et réseaux touristiques doivent surmonter ces problèmes et faire face aux défis; sans quoi ils connaîtront de nombreux dysfonctionnements aboutissant à une inefficacité croissante.

\section{Conclusions: facteurs cruciaux et suggestions}

Les clusters et réseaux examinés semblent capables de faciliter la procédure d'innovation des entreprises du tourisme rural et de contribuer au développement régional, à travers les résultats simples qui émanent des activités de clusters. Nous estimons que les facteurs cruciaux pour la réussite et susceptibles d'influencer l'efficacité des clusters / réseaux sur le niveau de destination locale et dans le contexte du tourisme rural doivent être les suivants:

- Une plate-forme commune d'intérêts devrait être établie et une structure organisationnelle claire et précise de cluster (relations, rôles et responsabilités des partenaires) est nécessaire.

- Les traits des partenaires en termes d'expertise, de professionnalisme et d'aptitude au networking ont des ramifications importantes sur la cohésion du cluster et le développement et la formation des conceptions et opinions communes, ainsi que sur la perception et l'appréhension des problèmes. L'engagement partagé, l'action collective et la continuité devraient être les caractéristiques vitales du cluster.

- Il est indispensable de considérer également les relations informelles parmi les partenaires.

- Les avantages géographiques du cluster ont des effets significatifs sur la collaboration, l'harmonie, le leadership et la fixation d'un agenda au sein du cluster. 
- $\quad$ La gestion de clusters locaux constitue un enjeu crucial ; il est impératif d'avoir un leadership dans l'industrie et une implication plus large de la population locale et des règles de conduite claires.

- Il est impératif d'avoir un intérêt orienté sur le client, relié par le management de connaissance et guidé par l'innovation et le service de qualité. Cette tâche semble être le plus grand défi de l'industrie du tourisme rural.

- La segmentation de clientèle basée sur des enquêtes est une première étape essentielle pour obtenir une gestion et un marketing efficaces dans le contexte du tourisme rural. En outre, des enquêtes de marché très améliorées sont nécessaires afin de fournir des informations de gestion.

- Une approche stratégique de la formation du cluster est indispensable, afin de garantir avec sérieux et cohérence des normes plus élevées de prestations et de produits, et d'être plus attractifs et compétitifs auprès de clients toujours plus exigeants.

- Il est essentiel d'avoir une participation mixte, d'obtenir l'implication tant du secteur public que du secteur privé. Il semble que la voie optimale pour une gestion et un marketing efficaces et cohérents se trouve quelque part entre la responsabilité et l'orientation vers les ressources du secteur public et la responsabilité de l'actif, et l'orientation vers le marché du secteur privé.

- $\quad$ Il est également primordial de préserver et de renforcer les qualités intrinsèques et l'identité distincte du site choisi dans les destinations, tant pour son propre compte que comme un élément essentiel d'attractivité pour les visiteurs.

On pourrait suggérer que le clustering / networking constitue une modalité fournissant des opportunités pour un changement et pour une énergie innovatrice. Les activités de cluster devraient donc être envisagées comme une condition préalable pour les politiques du tourisme rural. Dans les cas où ce dispositif est mis 
en application de manière appropriée, en tenant compte de tous les facteurs cités cidessus, il pourrait avoir une contribution significative dans la compétitivité de l'industrie du tourisme rural. Cette dernière semble contrainte à adopter des pratiques, dispositifs et outils tels que le clustering et networking pour parvenir à maturité. Le but étant, pour l'industrie du tourisme, de gagner son pari sur l'avenir: s'adapter à un monde nouveau, globalisé et exigeant. Ces alliances d'entreprises pourraient fonctionner plus efficacement à l'échelle de la destination locale, en adoptant une approche intégrale : à savoir, envisager et analyser les facteurs du développement économique local (Hall 2005), par exemple: (a) le maximum de partenaires locaux doivent être connectés (c'est-à-dire réseaux de marketing et clusters de gestion); (b) attirer des ressources externes (c'est-à-dire financement, aptitudes, savoir-faire et technologie); et (c) parvenir à acquérir des visiteurs en dehors de la région ( $c^{\prime}$ est-à-dire usage des TIC).

Cependant, on ne saurait omettre que notre analyse comporte certaines limitations. Il s'agit d'une étude exploratoire et les conclusions formulées ne peuvent en aucun cas être généralisées à tout projet particulier ou zone géographique. Des enquêtes et études empiriques sont nécessaires afin de la rendre plus pertinente. La méthodologie appliquée est celle de l'étude de cas et il faut la mettre à l'épreuve dans d'autres cas également afin de confirmer les facteurs cruciaux mis en évidence. L'étude présente a tenté de décrire la structure et d'analyser les activités de clusters touristiques développés en milieu rural. Des travaux empiriques plus étendus sont nécessaires pour examiner toutes les dimensions et caractéristiques de clusters d'entreprises. Ainsi, il semblerait nécessaire d'appréhender la dynamique des clusters et réseaux et de développer les stratégies adéquates pour leur gestion. 


\section{Bibliographie}

BERNINI, C. (2009): "Convention industry and destination clusters: Evidence from Italy" dans la Revue Tourism Management. In Press. Corrected Proof. Available online 13 January 2009

BÉTEILLE, R. (2000): "Le tourisme vert" dans la Collection «Que sais-je», no 3124,

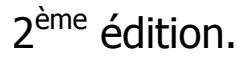

BUHALIS, D. (2005): "Information and communication technologies for tourism" dans PENDER, L. et SHARPLEY, R. (eds): The Management of Tourism. SAGE Publications. Londres.

CANARD, F. et RAVIX, J.-T. (2003): "Qualités subjective et objective dans le tourisme: des labels au partenariat" dans SPINDLER, J. et DURAND, $\mathrm{H}$. (éd.): Le tourisme au XXIe siècle. L'Harmattan. Paris.

CHASPOUL, C. (2000): "Les marques, un enjeu important pour le secteur du tourisme" dans Marques et labels touristiques dans Les Cahiers de I'AFIT.

EUROPEAN COMMISSION (2003): "Final report of the expert group on enterprise clusters and networks". Disponible en: www.competitiveness.org/article/archive/14/. Accedé le 28 de Novembre de 2008.

FYALL, A. et GARROD, B. (2005): Tourism Marketing: A Collaborative Approach. Channel View Publications. Clevedon.

GETZ, D., ANDERSSON, T. et LARSON, M. (2007): "Festival stakeholder roles: concepts and case studies" dans la Revue Event Management 10 (2-3).

GETZ, D. et BROWN, G. (2006): "Critical success factors for wine tourism regions: a demand analysis" dans la Revue Tourism Management, 27 (1).

HALL, C.M. (2005): "Rural wine and food tourism cluster network development" dans HALL, D.; KIRKPATRICK, I. et MITCHELL, M. (Eds), Rural tourism and sustainable business. Channel View Publications, Clevedon. 
HALL, C.M. et SHARPLES, L. (2008a): "Food Events and the Local Food System: Marketing, Management and Planning Issues" dans HALL, C. M. et SHARPLES, L. (Eds): Food \& Wine Festivals and Events around the World. Butterworth-Heinemann, Oxford.

HALL, C.M. \& Sharples, L. (2008b): "Future Issues and Trends: Food Events, Festivals and Farmers' Markets" dans HALL, C.M. et SHARPLES, L. (Eds): Food \& Wine Festivals and Events around the World. Butterworth-Heinemann. Oxford.

HITZ, M.; SIGALA, M. et MURPHY, M. (2006): Information and Communication Technologies in Tourism. Springer, New York \& Wien.

KOKKONEN, P. et TUOHINO, A. (2007): "The challenge of networking: Analysis of innovation potential in small and medium-sized tourism enterprises" dans la Revue The International Journal of Entrepreneurship and Innovation, 8 (1).

LAZZERETTI, L. et PETRILLO, C. (Editors) (2006): "Tourism Local Systems and Networking Advances" dans la Revue Tourism Research (series). Elsevier. Oxford.

LEMMETYINEN, A. et GO, F. (2009). "The key capabilities required for managing tourism business networks" dans la Revue Tourism Management, 30 (1).

MACKELLAR, J. (2006): "Conventions, festivals, and tourism: exploring the network that bids" dans la Revue Journal of Convention and Event Tourism, 8 (2).

MARSH, D. (1998): Comparing policy networks. Open University Press. Buckingham.

MICHAEL, E. J. (2003): "Tourism micro-clusters" dans la Revue Tourism Economics, 9 (2).

MIDDLETON, V.T.C. et HAWKINS, R. (2002): Sustainable Tourism: A Marketing Perspective ( $3^{\text {rd }}$ edn). Butterworth-Heinemann. Oxford.

NAISBITT, J. (1994) : Global Paradox. Nicolas Brealey, Londres.

NOVELLI, M. ; SCHMITZ, B. et SPENCER, T. (2006): "Networks, clusters and innovation in tourism: A UK experience" dans la Revue Tourism Management, 27 (6).

POON, A. (2002): Tourism, Technology and Competitive Strategies (4th edn). CAB International. Oxford.

PORTER, M. (1998): On Competition: A Harvard Business Review Book. Harvard Business School Publishing. Boston. 
PUF, Paris et BHAT, S. (2004): "The role and impact of strategic alliances and networks" dans "Destination marketing: the development of www.purenz.com" dans la Revue International Journal of Tourism Research, 6 (4).

ROBERTS, L. et HALL, D. (Eds) (2001): Rural Tourism and Recreation: Principles to Practice. CABI Publishing. Wallingford.

SAXENA, G. (2005): "Relationships, networks and the learning regions: Case evidence from the Peak District National Park" dans la Revue Tourism Management 26 (2).

SHARPLEY, R. (2005): "Managing the countryside for tourism: a governance perspective" dans PENDER, L. et SHARPLEY, R. (Eds): The Management of Tourism. SAGE Publications. London.

SOTERIADES, M. et AVGELI, V., (2007): "Promoting tourism destinations: a strategic marketing approach" dans la Revue Tourism: an international interdisciplinary journal, 55 (3).

SOTERIADES, M. et VARVARESSOS, S. (2002): "Rural tourism's planning into the framework of European initiative Leader Plus" dans Iña Revue Archives of Economic History, XIV (2).

SPINDLER, J. (2003): "Introduction" dans SPINDLER, J. et DURAND, H. (éd.), Le tourisme au XXI siècle. L'Harmattan. Paris.

STOKES, R. (2006): "Network-based strategy making for events tourism" dans la Revue European Journal of Marketing, 40 (5-6).

TINSLEY, R. et LYNCH, P. (2007): "Small business networking and tourism destination development: A comparative perspective" dans la Revue The International Journal of Entrepreneurship and Innovation, 8 (1).

WILSON, S.; FESENMAIER, D.; FESENMAIER, J. et VAN ES, L. (2001): "Factors for success in rural tourism development" dans la Revue Journal of Travel Research, 40 (2).

YIN, R. M. (2003): Case Study Research. Design and Method (3rd edn). Sage Publications. Thousand Oaks. 


\section{Webgrafía}

www.anher.gr

www.wineroads.gr

www.eurogites.org

www.guestinn.com

www.idinet.gr

\section{Annexe}

Carte no 1: Grèce et Crète

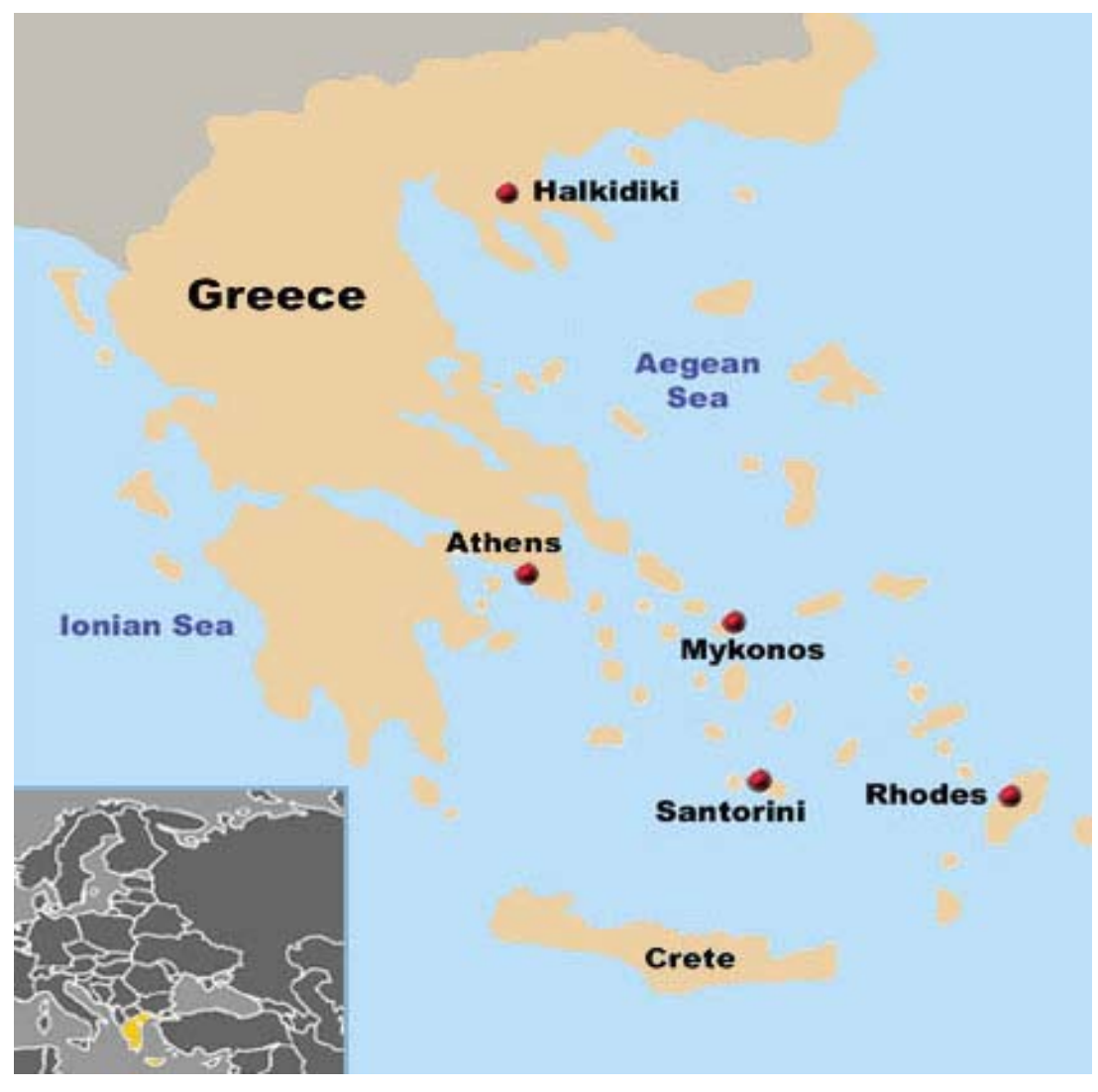


Soteriades, Marios (2010): Clusters et reseaux dans le cadre du tourisme rural: l'experience grecque. $N^{\circ}$ 23. Noviembre. Año XIV. Páginas: 85-117

\section{CRETE}

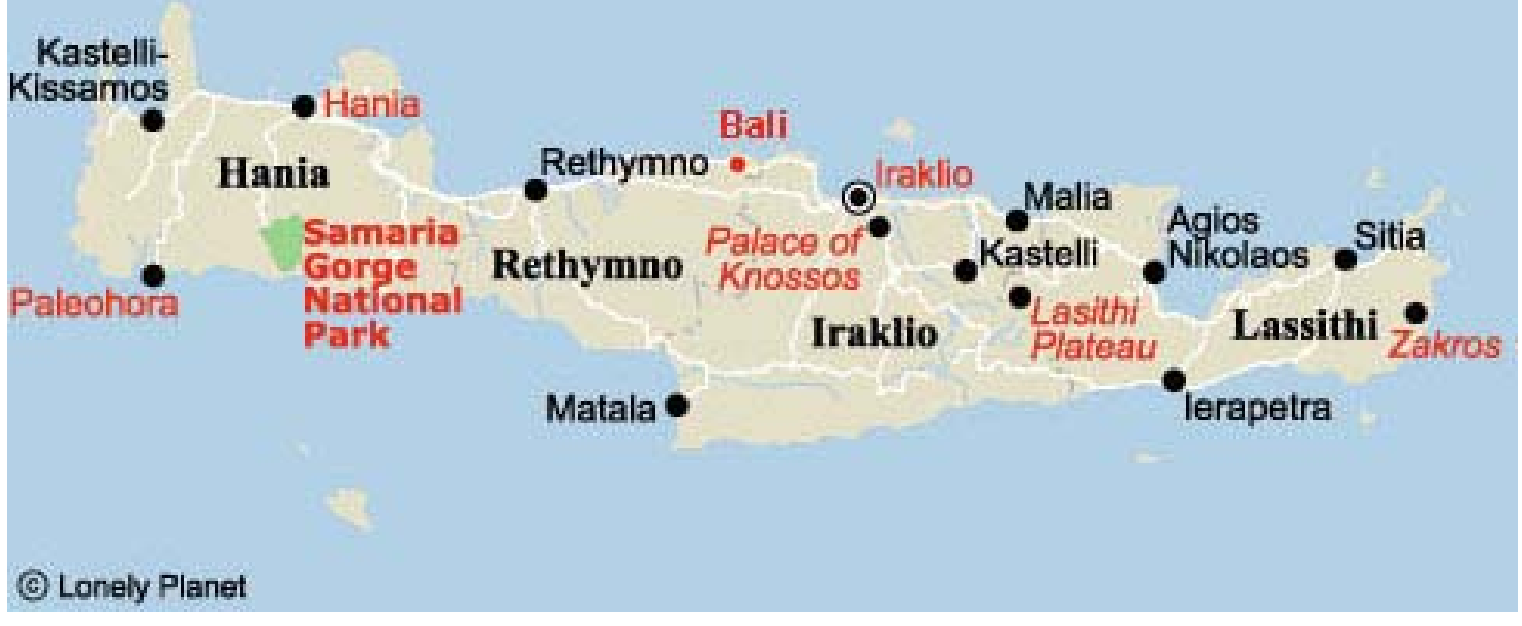

Sources: http://maps.google.com/maps et http://www.thehotel.gr/map-of-crete/

\section{Carte no 2 : Routes des Vins de la Grèce du Nord}

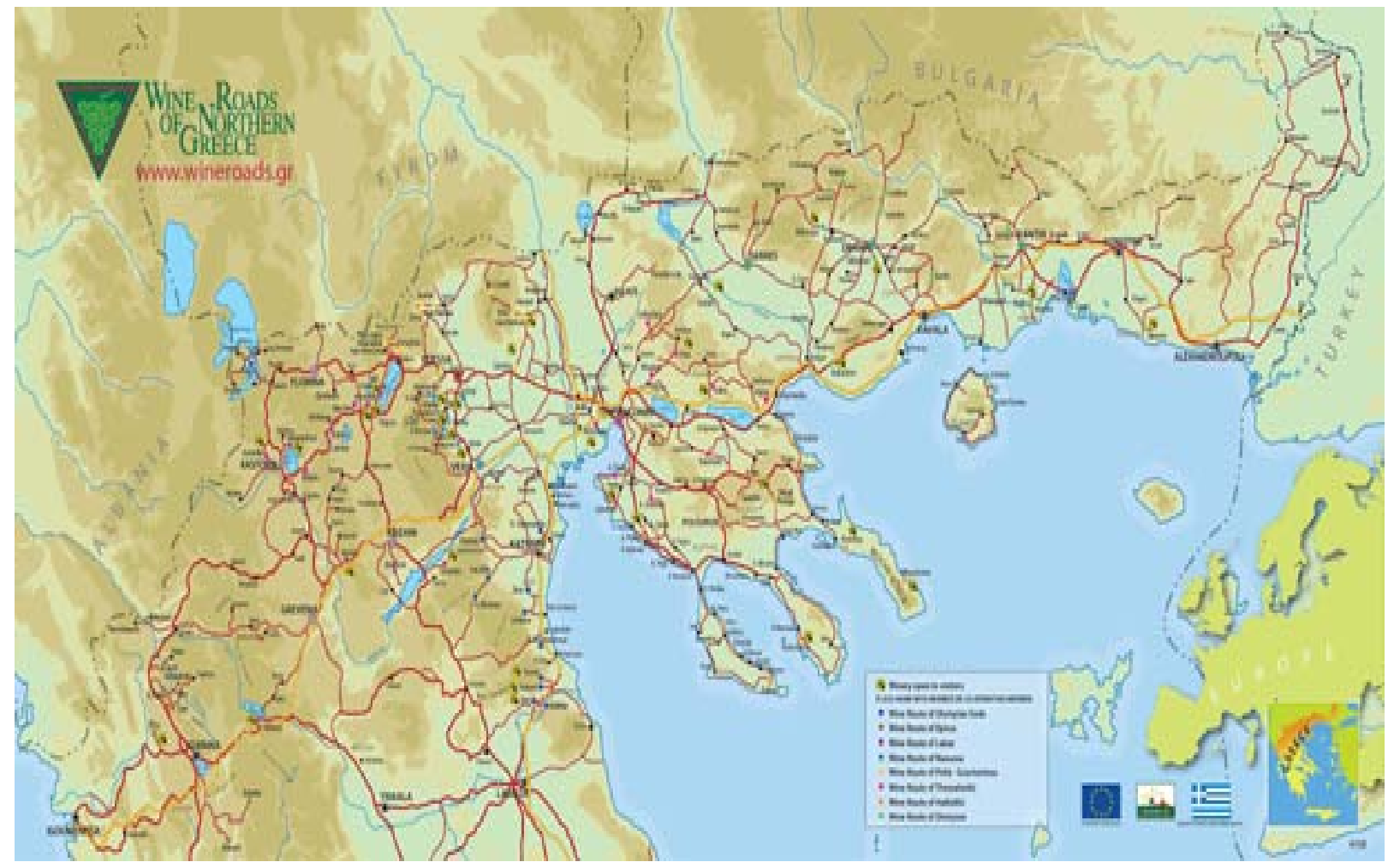

Source: www.wineroads.gr/eng/index.php\# 